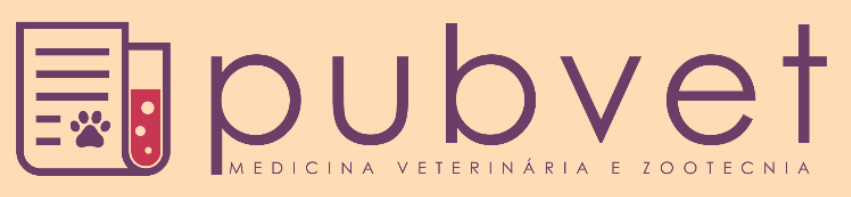

https://doi.org/10.31533/pubvet.v13n11a448.1-4

\title{
Carcinoma prostático e urocistolitos concomitantes em cão Dálmata
}

\author{
Talita Wajczyk $^{1} \oplus$, Rodrigo Von Mühlen ${ }^{2} \bullet$, Laísa Gabriela Cunha ${ }^{2}$, Marisa Ometto ${ }^{3}$, Juliana de \\ Abreu Pereira $^{4 * \bullet}$, Katherinne Barth Wanis Figueiredo ${ }^{10}$, Ana Cristina Almeida ${ }^{1}{ }^{\circ}$ \\ ${ }^{I}$ Graduanda em Medicina Veterinária da Sociedade Educacional de Santa Catarina (Unisociesc). \\ ${ }^{2}$ Médico(a) veterinário na clínica VetCenter, Joinville - SC Brasil. \\ ${ }^{3}$ Médica Veterinária autônoma atuante na área de ultrassonografia veterinária, Joinville - SC Brasil \\ ${ }^{4}$ Docente do curso de Medicina Veterinária da Sociedade Educacional de Santa Catarina (Unisociesc), Departamento de Medicina \\ Veterinária, Joinville - SC Brasil. \\ *Autor para correspondência. E-mail: juliana.abreu@unisociesc.com.br
}

\begin{abstract}
Resumo. A neoplasia prostática é uma das raras afecções que acometem cães machos, adultos ou geriátricos não castrados, reduzindo a qualidade de vida e acarretando piora clínica. $\mathrm{O}$ presente relato discorre sobre a neoplasia prostática concomitante a urolitíase, os sinais clínicos dessa enfermidade, o diagnóstico auxiliado por exame ultrassonográfico e citológico de material coletado durante o transcirúrgico de uma cistotomia em um Dálmata adulto e castrado.
\end{abstract}

Palavras chave: canino, neoplasia, próstata, urolitíases

\section{Prostactic carcinoma and urocystolites concomitant in a Dalmatia dog}

\begin{abstract}
The prostatic neoplasia is one of rare disease that affect male dogs, adult or geriatrician non castraded, reducing the quality of life and leading to clinical worsening. The present paper discourse about the prostatic neoplasia concomitant to a urolithiasis, clinical signs of this diseases, the diagnosis assisted by ultrassonographic and cytological exams collected during the trans-surgical of a cystotomy of a male non castraded Dalmatian dog.
\end{abstract}

Keywords: canine, neoplasia, prostate, urolithiasis

\section{Carcinoma prostático y urolitocitos concomitantes en perro Dálmata}

Resumen. La neoplasia prostática es una de las raras enfermedades que afectan a los perros machos, adultos o geriátricos no castrados, lo que reduce la calidad de vida y provoca un empeoramiento clínico. El presente caso analiza la neoplasia prostática concomitante de urolitiasis, los signos clínicos de esta enfermedad, el diagnóstico con ayuda de ultrasonido y el examen citológico del material recogido durante la cistotomía de un Dálmata adulto castrado.

Palabras clave: neoplasia, perro, próstata, urolitiasis

\section{Introdução}

A glândula prostática canina pode ser acometida por diversas afecções, principalmente em adultos e idosos não castrados, em função da disposição anatômica entre os órgãos do sistema urogenital (Purswell et al., 2000). As enfermidades descritas de maior incidência são a hiperplasia prostática benigna, cuja casuística é maior que 95\% (Gobello \& Corrada, 2002; Smith, 2008), prostatite aguda (Smith, 2008); cistos prostáticos, com incidência de 14\% nas raças grandes (Smith, 2008), abscessos prostáticos (Smith, 2008) e neoplasias.

As neoplasias são consideradas raras, com frequência de 5 a 7\% (Memon \& Mickelsen, 2004; Smith, 2008). Quando diagnosticadas, são comumente observadas em cães adultos e idosos (Alves et al., 2010), 
sendo 10 anos a média de idade do animal no momento do diagnóstico. Dentre os tipos mais comuns de neoplasias encontradas citam-se o adenocarcinoma e o carcinoma indiferenciado (Johnston et al., 2000; Purswell et al., 2000; Smith, 2008). No exame clínico questiona-se o padrão urinário e de fezes, verificando a ocorrência de sinais como tenesmo e hematúria. Avaliam-se nessa glândula alterações quanto ao seu tamanho, forma, simetria e evidência de dor à palpação.

Quando diagnosticada a neoplasia, o tratamento apresenta prognóstico desfavorável. Usualmente esses animais estão em estágios avançados da doença e, portanto, com limitações na escolha de tratamentos (Smith, 2008). As terapias que podem ser empregadas para estes pacientes são a radioterapia, para a redução de tamanho da glândula; e a intervenção cirúrgica, mas com probabilidade de complicações, a citar a incontinência urinária (Smith, 2008).

$\mathrm{O}$ presente relato teve por objetivo descrever a ocorrência concomitante do carcinoma prostático e urocistólitos em um paciente canino da raça dálmata, bem como os métodos empregados no diagnóstico e tratamento para o animal enfermo.

\section{Relato de caso}

Um Dálmata, castrado, 8 anos e peso de 24 quilos foi atendido em uma clínica em Santa Catarina. O tutor relatou mudanças no comportamento como tremores, prostração, mobilidade e alimentação diminuídas, abdômen rígido e diarreia, iniciados cinco e três dias antes do atendimento, respectivamente. Por cogitar a ingestão de corpo estranho medicou por conta paracetamol.

Ao exame físico o paciente manifestou hematúria e limitação na micção. Amostras de sangue para exames de hemograma e bioquímica sérica foram coletadas e analisadas ${ }^{1}$. O hemograma estava normal (Tabela 1) e o bioquímico revelou extrapolação do limite superior da globulina, um indicativo de hipergamaglobulinemia. A razão albumina/globulina 0,65 está próxima do limite inferior e passível de associação às alterações do sistema imune.

Tabela 1. Resultado dos exames laboratoriais.

\begin{tabular}{|c|c|c|c|c|c|c|c|}
\hline Parâmetro & Valor & Referência & Unidade & Parâmetro & Valor & Referência & Unidade \\
\hline Eritrócitos & 6,0 & $5,5-8,5$ & $\mathrm{M} / \mu \mathrm{L}$ & \multirow{2}{*}{$\begin{array}{l}\text { Creatinina } \\
\text { NUP }^{3}\end{array}$} & 0,6 & $0,5-1,8$ & \multirow{2}{*}{$\mathrm{mg} / \mathrm{dL}$} \\
\hline Reticulócitos & 69 & $10-110$ & \multirow{4}{*}{$\mathrm{K} / \mu \mathrm{L}$} & & 9 & $7-27$ & \\
\hline \multirow[t]{2}{*}{ Plaqueta } & \multirow[t]{2}{*}{348} & \multirow[t]{2}{*}{$175-500$} & & Albumina & 3 & $2,3-4,0$ & $\mathrm{~g} / \mathrm{dL}$ \\
\hline & & & & Globulina & 4,6 & $2,5-4,5$ & - \\
\hline Leucócitos & 13,2 & $5,5-16,9$ & & ALT & 34 & $10-125$ & $\mathrm{U} / \mathrm{L}$ \\
\hline Neutrófilo & 69,8 & \multirow{5}{*}{$* 2$} & \multirow{5}{*}{$\%$} & Neutrófilo & 9 & $2-12$ & \multirow{5}{*}{$\mathrm{K} / \mu \mathrm{L}$} \\
\hline Linfócito & 14,6 & & & Linfócito & 1,9 & $0,5-4,9$ & \\
\hline Monócito & 11,5 & & & Monócito & 1,5 & $0,3-2,0$ & \\
\hline Eosinófilo & 3,9 & & & Eosinófilo & 0,51 & $0,10-1,49$ & \\
\hline Basófilo & 0,2 & & & Basófilo & 0,03 & $0,0-0,1$ & \\
\hline
\end{tabular}

${ }^{1}$ Amostras de sangue para exames de hemograma e bioquímica sérica foram coletadas e analisadas nos aparelhos LaserCyteDx e CatalystOne, ${ }^{2}$ Apenas valores de referências informados pelos aparelhos LaserCyteDx e CatalystOne foram reportados. NUP ${ }^{3}$

No ultrassom foi descrito paredes hipoecogênicas e espessadas no estômago com até $0,76 \mathrm{~cm}$, sugerindo injúria gástrica. O linfonodo ilíaco medial direito era ecogênico e aumentado $(2,80 \mathrm{~cm}$ por $1,17 \mathrm{~cm}$ ). As paredes da vesícula urinária eram irregulares e ecogênicas, indicando injúria vesical ou cistite. Na figura 1 nota-se o conteúdo heterogêneo da vesícula, com formas ovaladas (medindo até 1,54 $\mathrm{cm}$ no maior eixo) e contornos irregulares, hiperecóicas e produtoras de sombra acústica posterior, compatíveis com urolitíases. Sedimento urinário de células e cristais com urólitos em formação no lúmen vesical eram visíveis.

A próstata exibia topografia habitual e dimensões normais, medindo $3,91 \mathrm{~cm}$ por $3,93 \mathrm{~cm}$ com contornos irregulares, ecogenicidade mista, ecotextura heterogênea com focos de mineralização com até $0,65 \mathrm{~cm}$ no maior eixo e pontos hiperecóicos dispersos pelo parênquima. Os achados são exibidos na figura 1 e sugeriram prostatite com possível neoplasia prostática. 
O cão foi internado e recebeu fluidoterapia com solução Ringer Lactato à taxa de $60 \mathrm{~mL} / \mathrm{h}$ nesse período. Foram administrados por via endovenosa: Dipirona $25 \mathrm{mg} / \mathrm{kg}$ e Tramadol $4 \mathrm{mg} / \mathrm{kg}$ a cada 12 horas; e Enrofloxacina $5 \mathrm{mg} / \mathrm{kg}$, Omeprazol $1 \mathrm{mg} / \mathrm{kg}$ e Meloxicam $0,1 \mathrm{mg} / \mathrm{kg}$ a cada 24 horas, mantidos até $\mathrm{o}$ ato cirúrgico.

$\mathrm{Na}$ cistotomia foi passada uma sonda uretral número 8 para evitar a repleção vesical, mantida por cinco dias. Na sua passagem não foram percebidos cálculos na uretra. A parede da vesícula estava inflamada e friável. Urólitos de dimensões variáveis e coloração esverdeada foram removidos (Figura 2). Urinálise e análise química dos urólitos não foram autorizados pelo proprietário.

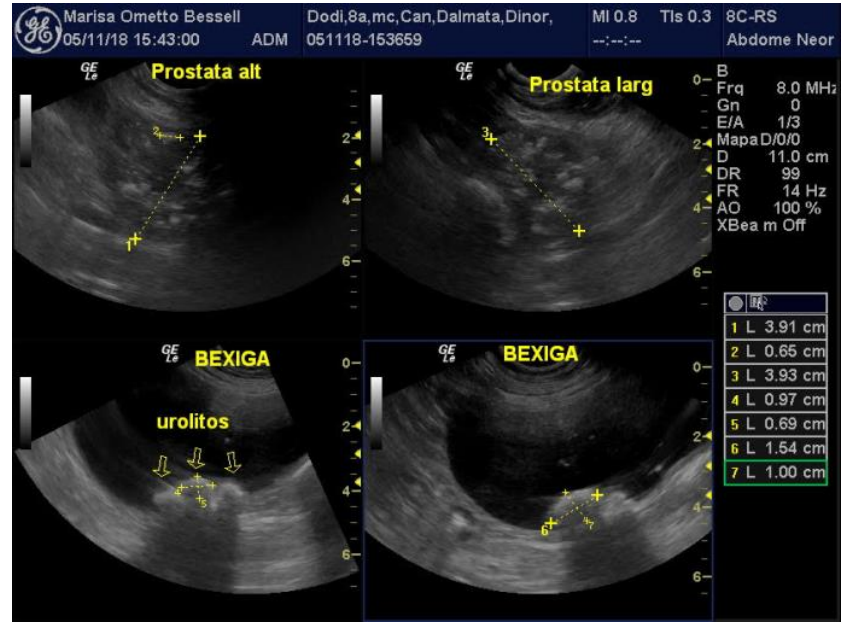

Figura 1. Imagens ultrassonográficas de vesícula urinária e próstata de dálmata acometido por carcinoma prostático.

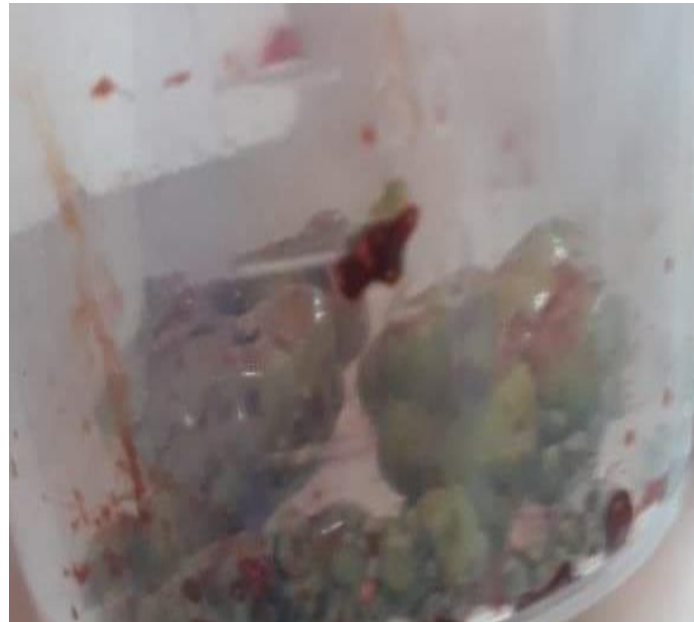

Figura 2. Urólitos removidos na cirurgia.

No transcirúrgico, amostras da próstata foram coletadas para citologia por punção aspirativa com agulha fina. Esta exibiu alta celularidade com $3 \%$ de células inflamatórias, células neoplásicas de origem epitelial glandular; dispostas em arranjos glandulares e moderadamente pleomórficas (Figura 3A), com núcleo redondo a oval, bi e tri nucleação (Figura 3B), cromatina frouxa, nucléolos únicos e/ou múltiplos, por vezes pleomórficos; citoplasma basófilo, redondo a poligonal, moderadamente delimitado. Foram observadas células de núcleo nu, citomegalia e cariomegalia (Figura 3C). Anisocitose e anisocariose eram marcantes. O fundo da lâmina continha hemácias, plaquetas e material basófilo amorfo; compatível com necrose, comum em aspirados de neoplasias malignas. Os achados sugeriram carcinoma prostático. Exame histopatológico para a corroboração foi negada pelo tutor, o qual foi orientado a uma consulta oncológica e a proceder com prostatectomia, porém declinou em ambos.

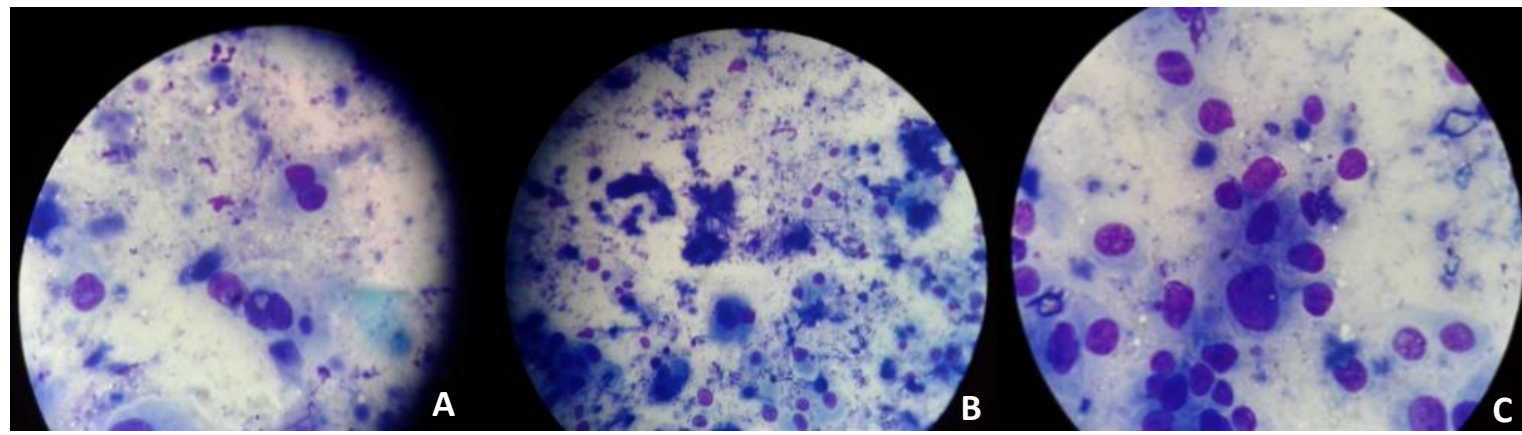

Figura 3. Citologia da próstata: (A) células com núcleos pleomórficos, (B) multinucleação, (C) citomegalia e cariomegalia.

O cão ficou internado após a cirurgia por cinco dias mantendo a prescrição do pré-cirúrgico além de carprofeno em comprimido $4,16 \mathrm{mg} / \mathrm{kg}$ administrado por via oral a cada 24 horas. A prescrição da alta médica consistiu nas seguintes medicações na apresentação em comprimidos: enrofloxacina $6,25 \mathrm{mg} / \mathrm{kg}$ por 7 dias e carprofeno $4,4 \mathrm{mg} / \mathrm{kg}$ por 4 dias, ambos a cada 24 horas; e dipirona $20,83 \mathrm{mg} / \mathrm{kg}$ a cada 12 horas por 4 dias, todas administradas por via oral. A retirada dos pontos ocorreu seis dias após alta. 


\section{Discussão}

A neoplasia prostática é uma das raras afecções que acometem cães machos, adultos ou geriátricos não castrados, reduzindo a qualidade de vida.

O exame de imagem e a citologia foram conclusivos como descrito por Russo et al. (2009) e Galvão et al. (2011), os quais reforçam ainda os sinais clínicos, as alterações percebidas na palpação retal e no exame histológico para o diagnóstico de neoplasia prostática. Para LeRoy \& Northrup (2009) disúria, hematúria, disquesia, ataxia, letargia, anorexia e perda de peso são sinais evidentes. Destes poucos eram notados no cão e concomitantes com o desconforto da urolitíase. No estudo de Bradbury et al. (2009) $67 \%$ dos cães com evidência de mineralização $(n=5)$ ao ultrassom apresentavam neoplasia prostática, sendo este achado presente neste relato.

A cistotomia atuou no alívio ao urinar e na hematúria. As dimensões dos urólitos obtidas na imagem contraindicam o tratamento clínico por restrição proteica, alteração do $\mathrm{pH}$ urinário ou inibição da xantina-oxidase.

\section{Conclusão}

Neoplasias prostáticas, mesmo com baixa casuística, ocorrem na rotina clínica. Como discorrido nesse relato, pode não ser a queixa principal do tutor. $\mathrm{O}$ exame de imagem e a citologia foram conclusivos.

\section{Referências bibliográficas}

Alves, C. E. F., Faleiro, M. B. R., Amorim, R. L. \& Moura, V. M. B. D. (2010). Avaliação histológica da próstata de cães adultos sexualmente intactos. Arquivo Brasileiro de Medecina Veterinária e Zootecnia, 62(3):596-602.

Bradbury, C. A., Westropp, J. L. \& Pollard, R. E. (2009). Relationship between prostatomegaly, prostatic mineralization, and cytologic diagnosis. Veterinary Radiology \& Ultrasound, 50(2):167171.

Galvão, A. L. B., Ferreira, G. S., Léga, E., Costa, P. F., Ondani, A. C. \& Denicol, A. (2011). Principais afecções da glândula prostática em cães. Revista Brasileira de Reprodução Animal, 35(4):456-466.

Gobello, C. \& Corrada, Y. (2002). Noninfectious prostatic diseases in dogs. Compendium on Continuing Education for the Practising Vegeterian-North, 24(2):99-109.

Johnston, S. D., Kamolpatana, K., Root-Kustritz, M. V. \& Johnston, G. R. (2000). Prostatic disorders in the dog. Animal Reproduction Science, 60405-415.

LeRoy, B. E. \& Northrup, N. (2009). Prostate cancer in dogs: comparative and clinical aspects. The Veterinary Journal, 180(2):149-162.

Memon, M. A. \& Mickelsen, W. D. (2004). Distúrbios hereditários e congênitos dos sistemas reprodutivos do macho e da fêmea. In S. J. Ettinger \& E. C. Feldman (Eds.), Tratado de medicina interna veterinária doenças do cão e do gato. (pp. 1668-1669). São Paulo, São Paulo, Brasil: Guanabara Koogan.

Purswell, B. J., Parker, N. A. \& Forrester, S. D. (2000). Prostatic diseases in dogs: a review Veterinary of Medicine315-321.

Russo, M., Vignoli, M., Catone, G., Rossi, F., Attanasi, G. \& England, G. C. (2009). Prostatic perfusion in the dog using contrast-enhanced Doppler ultrasound. Reproduction in Domestic Animals, 44334335 .

Smith, J. (2008). Canine prostatic disease: a review of anatomy, pathology, diagnosis, and treatment. Theriogenology, 70(3):375-383.

Recebido: 12 de agosto, 2019.

Aprovado: 2 de novembro, 2019.

Publicado: 07 de dezembro, 2019.

Licenciamento: Este artigo é publicado na modalidade acesso aberto sob a licença Creative Commons Atribuição 4.0 (CC-BY 4.0), a qual permite uso irrestrito, distribuição, reprodução em qualquer meio, desde que o autor e a fonte sejam devidamente creditados. 